\title{
Optimal design of biomass supply chains with integrated process design
}

\author{
Theodoros Damartzis*, François Maréchal \\ Industrial Process and Energy Systems Engineering (IPESE), Ecole Polytechnique \\ Fedérale de Lausanne (EPFL) Valais, 1951, Sion, Switzerland
}

theodoros.damartzis@epfl.ch

\begin{abstract}
The current work focuses on the optimal design of biomass supply chains, addressing the conversion of raw materials into useful services. The latter include not only heat and power but also synthetic bio-based fuels (e.g. bio-SNG). Optimally pre-designed processing units representing the conversion facilities responsible for the transformation of biomass are employed. This permits the use of standardized units that act as building blocks within a MILP optimization problem, significantly simplifying the formulation and solution of the problem. These building blocks encompass a variety of transformation options and carry specific information regarding their capacity, total processing cost, overall efficiency and energetic performance. Then, the supply chain design optimization problem aims not only to identify the most suitable conversion plant type but also to determine their location by minimizing the overall cost, expressed as the capital and operating expenses of the processing units as well as the transport costs between the points of operation. The above formulation is applied for the conversion of animal manure to different end products in the district of Brig in southern Switzerland. Results reveal the predominance of large centralized bio-SNG producing units as a consequence of their higher conversion efficiency.
\end{abstract}

Keywords: supply chain design, bio-SNG, biomass conversion, animal manure.

\section{Introduction}

The increasing depletion of the fossil-based energy resources as well as the new mandates for the insertion of renewables in the global energy mix are continuously shaping the background for the energy transition. As renewable energy sources are presented to be promising alternatives to the traditional fossil resources, an intensification of the efforts to deploy sustainable biomass-based energy systems is ongoing not only from a strategic decision point of view, but also from an environmental perspective (d'Amore and Bezzo, 2016).

However, the introduction of bioenergy into the existing energy market necessitates not only the efficient design of the processing of raw materials but also the consideration of the spatial interactions of the conversion plants with the supply and demand points (Chomette et al., 2018). A number of recent studies address the issue of the biomass supply chain design. Costa Melo et al. (2018) present a data envelopment analysis approach (DEA) to identify the most suitable transport pathway of wood from Africa to Europe. How and Lam (2017) proposed a novel optimization approach that incorporating principal component analysis (PCA) and analytical hierarchy process 
(AHP) to determine the optimal transportation design and processing hub location in an integrated biomass supply chain. The work of Ghaderi et al. (2016) provides a comprehensive review regarding the design of biomass supply chains. In this paper, a large number of published works are compared and a large diversity on modeling approaches is revealed. Focusing more on manure as a source material and the production of bio-SNG, Calderón et al. (2017) provide a framework for the design and optimization of bio-SNG supply chain using av explicit MILP multi-period scheme.

However, one should not forget that the role of process design within the definition of the supply chain is crucial. Thus, a bigger approach is needed that accounts for the integration of process design with the supply chain, highlighting the benefits and revealing potential synergies between the various steps of the conversion path from raw materials to services. Moreover, such a formulation will set the transition from the wellestablished process design level to the larger scale market level. To account for the role of the design of the processing units in the supply chain, expressed both as the type of conversion plant but also in relation to the output capacity, the current work presents a holistic approach based on the model presented by Chomette et al. (2018) for the design of wet biomass supply chains. To this end, the type and size of the conversion plants will play a decisive role in the formulation of the supply chain. The type of the plant refers to the various sub-processes that are used to convert the raw material to useful products as well as the formulation of the entire process flowsheet, while the size represents the output capacity in energy units. Using optimally designed conversion plants as predefined building blocks permits the synthesis of the entire supply chain through a MILP optimization problem that aims to minimize the total cost, expressed as the sum of the processing and transport expenses. The above formulation is then presented in an illustrative example for Brig, one of the highest manure producing districts of the canton of Valais in Switzerland.

\section{Methodology}

\subsection{Manure conversion and design of the building blocks}

The use of animal manure as the raw biomass material offers the possibility of using a plethora of conversion options of variable efficiencies depending on the desired end use as well as the conversion pathway that is followed. The common conversion process is anaerobic digestion, in which manure is converted to biogas through a series of complex (bio-)chemical reactions. The employment of pretreatment options of the raw biomass offers the chance to increase the biogas yield from the digester, inducing however an extra processing cost. Especially in cases where thermal pretreatment is used, extra degrees of freedom appear in the design process, related to the heat exchange between the pretreatment unit and the rest of the process. Biogas, being a mixture of $\mathrm{CH}_{4}$ and $\mathrm{CO}_{2}$ in a molar ratio of roughly 60:40 has to undergo through a cleaning and upgrading process to remove traces of impurities like $\mathrm{H}_{2} \mathrm{~S}, \mathrm{NH}_{3}$, siloxanes etc. and increase its methane content, respectively. The final stream of upgraded biogas consists mainly of $\mathrm{CH}_{4}$ and can be used as bio-SNG for grid insertion or power production in engines or fuel cells. On the other hand, anaerobic digestion leaves an undigested stream that still contains a large portion of the initial carbon content. It is therefore of great interest to extract the latter in order to maximize the efficiency of the overall process. Hydrothermal gasification (HTG) offers the possibility of converting wet organic 
streams into methane using high pressure in a complex reactor scheme. The supercritical conditions that prevail within the HTG reactor ensure that the nitrous and phosphoric minerals are released unharmed in the residual output stream. Moreover, the use of catalytic methanation to convert the separated stream of $\mathrm{CO}_{2}$ from the biogas is another option of further increasing the final bio-SNG yield. The methanation reactor can be fed with hydrogen produced through the electrolysis of water using renewables (e.g. solar power).

Using the MILP optimization framework described in Vigot et al. (2018), a number of possible configurations have been designed, each emphasizing in different process schemes and end products. Figure 1 shows the pareto front correlating the annualized process cost (in Swiss francs) with the overall energy efficiency of the process for a 100 kW unit.

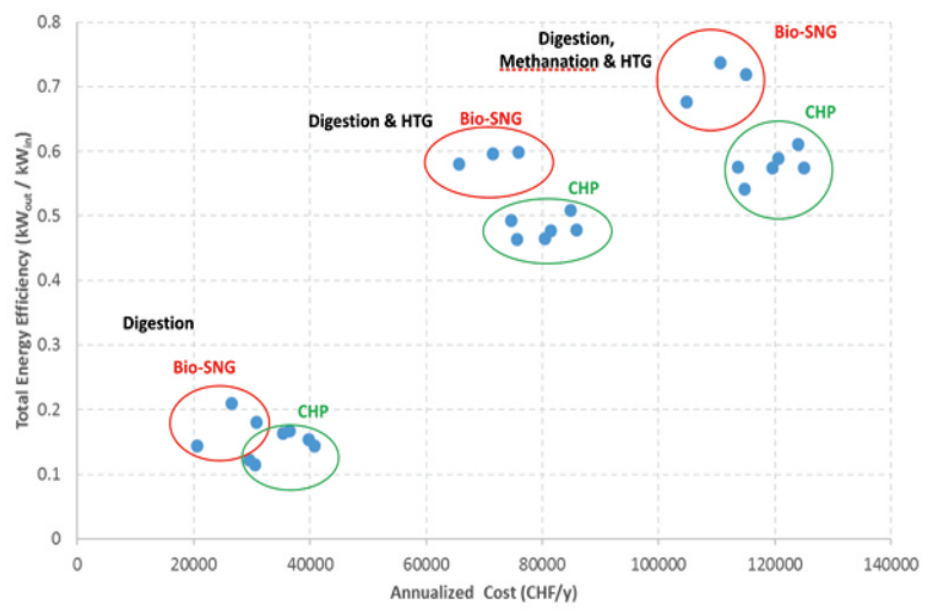

Figure 1. Pareto front of various process designs for manure conversion.

\subsection{Employed flowsheets and typical sizes}

Three different indicative flowsheets have been chosen to be used in the design of the supply chain, each focusing in a different end product. The employed flowsheets are depicted in Figure 2.

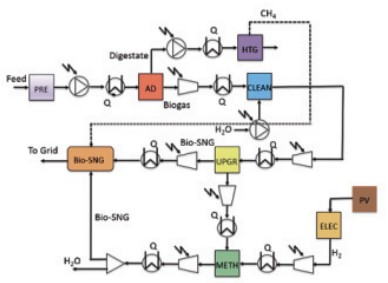

Bio-SNG

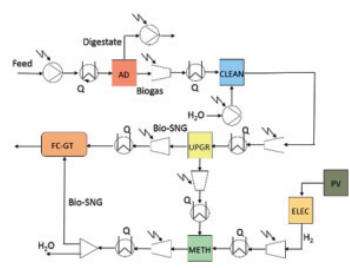

Power

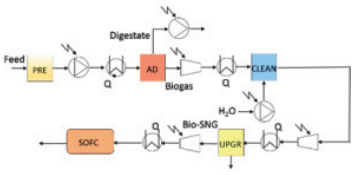

$\mathrm{CHP}$

Figure 2. Employed flowsheets for the conversion of manure to different end products. 
As already mentioned, the size of the conversion units plays an important part in the supply chain design. The specific costs of each flowsheet expressed as Swiss francs per $\mathrm{kW}$ produced is shown in Table 1. The typical sizes are selected with a reference on the raw material supply as well as the existing plants in the application area. The nonlinear dependence of the specific cost with the output capacity of each plant stems from the nonlinear dependence of the process efficiency with the plant dimensions.

Table 1. Specific costs (cost per kW) in Swiss Francs (CHF) of the employed flowsheets

\begin{tabular}{lrrr}
\hline & $50 \mathrm{~kW}$ & $100 \mathrm{~kW}$ & $250 \mathrm{~kW}$ \\
\hline Bio-SNG & 2945.90 & 2292.54 & 1830.96 \\
Power & 2188.55 & 1821.40 & 1605.95 \\
CHP & 866.28 & 709.39 & 594.17 \\
\hline
\end{tabular}

\section{Application - Results}

The three types of conversion plants presented in Section 2 together with the three typical sizes are used for the design of a supply chain for manure conversion in the commune of Brig in southern Switzerland. Six source sites have been identified and the corresponding manure potentials are taken from Thees et al. (2017). Using the design framework presented by Chomette et al. (2018), the results are illustrated in Figure 3.

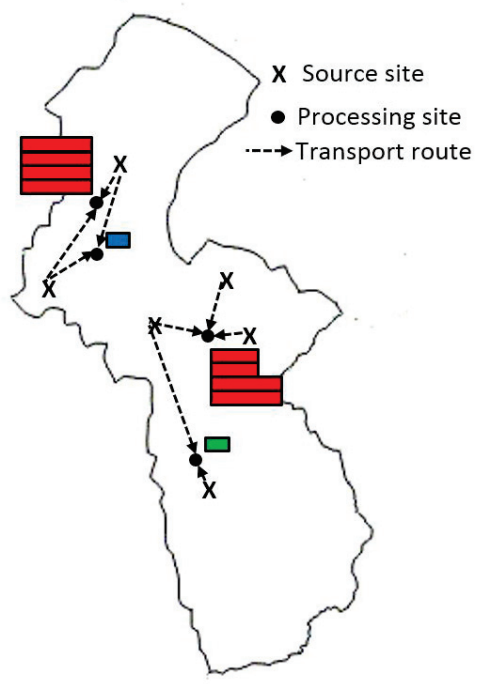

Figure 3. Supply chain results for manure conversion in the district of Brig, Switzerland 
The horizontal length of the bars representing the processing units corresponds to the energetic output and specifically, $50 \mathrm{~kW}$ for small bars, $100 \mathrm{~kW}$ for medium bars and $250 \mathrm{~kW}$ for large bars. Similarly, the colors of the units correspond to the type of the plant, namely red for bio-SNG producing plants, blue for power production and green for CHP (also see Figure 2). It is seen that large centralized bio-SNG units are favored over the rest of the options due to the much larger energy efficiency of the process. In other words, these plants are able to convert more effectively a larger capacity of raw materials into bio-SNG. The centralized fashion in the placement is a direct consequence of the larger capital expenses that correspond to this type of conversion (Table 2). On the other hand, one can see that one biomass source is not limited to feeding a single conversion unit as shown in the northern part of the commune. According to the results, heat and power producing plants act as a supplement to the residual manure that is more expensive to be converted to bio-SNG. It has to be noted that this of course depends also on the initial choice of the building blocks. In other words, if an exhaustive investigation was made using all the available conversion options, it would be apparent that the highly efficient designs would be preferentially used over the other options. This of course, will increase the accuracy of the results but also drastically raise the computational effort and is the next step of this research.

\section{Conclusions}

A study for the design of manure supply chains was presented here. Based on a previous work, the approach involves the use of pre-designed processing plants representing the main steps of the biomass-to-services conversion chain. Such plants come equipped with information on their assigned capacity and type, the first being expressed as the energetic output of the conversion process while the second as the pathway from raw materials to products and are then used within a MILP design optimization framework. The latter is able to perform the spatial allocation by selecting the most suitable conversion type and size of each plant with regard to the supply of raw biomass by minimizing the total cost of the supply chain represented by the sum of the processing and transport costs. Three pre-defined process flowsheets, each accounting for the conversion of manure to different end products and with a different energetic efficiency, as well as three typical plant sizes selected by considering the domain of application are used for the design of the manure supply chain for the district of Brig in Switzerland. According to the model outputs, the conversion to bio-SNG in large plants of centralized fashion seems to be the most economically viable option.

\section{Acknowledgements}

This research project is financially supported by the Swiss Innovation Agency Innosuisse and is part of the Swiss Competence Center for Energy Research SCCER BIOSWEET.

\section{References}

A. J. Calderón, P.Agnolucci, L. G. Papageorgiou, 2017, An optimisation framework for the strategic design of synthetic natural gas (BioSNG) supply chains, Applied Energy, 187, 929955.

G.A. Chomette, Th Damartzis, F. Maréchal, 2018, Optimal design of biogas supply chains, Computer Aided Chemical Engineering, 43(A), 669-674, 2018. 
I. Costa Melo, A. Rentizelas, A. J. Paulo Nocera, J.S. Campoli, D.A. do Nascimento Rebelatto, 2018, An assessment of biomass supply chain : a DEA application. Athens Journal of Sciences, 5 (2). pp. 125-140.

F. d'Amore, F. Bezzo, 2016, Strategic optimisation of biomass-based energy supply chains for sustainable mobility, Computers and Chemical Engineering 87, 68-81.

H. Ghaderi, M. S. Pishvaee, A. Moini, 2016, Biomass supply chain network design: An optimization-oriented review and analysis, Industrial Crops and Products, 94 (30), 972-1000.

B.S. How, H. L. Lam, 2017, Novel Evaluation Approach for Biomass Supply Chain: An Extended Application of PCA, Chemical Engineering Transactions, 61, 1591-1596.

O. Thees, V. Burg, M. Erni, G., Bowman, R. Lemm, 2017, Biomass potenziale der Schweiz für die energetisce Nutzung, WSL Berichte, Heft 57.

M.A. Vigot, Th. Damartzis, F. Maréchal, 2018, Thermoeconomic design of biomass biochemical conversion technologies for advanced fuel, heat and power production, Computer Aided Chemical Engineering, 44(A), 1801-1806, 2018. 